\title{
DESIGNING E- GEOGRAPHIC ATLAS OF THAI NGUYEN PROVINCE FOR SCHOOL GEOGRAPHY TEACHING
}

\author{
Hoang Thanh Van \\ Ph.D Program in Civil and Hydraulic Engineering, Feng Chia University, Taichung, Taiwan \\ st_van@gis.tw
}

KEY WORDS: Atlas paper, E-atlas, Thai Nguyen statistic book, geography local area, GIS

\begin{abstract}
:
The article deals with designing (Lam, 2003) and editing electronic geographical Atlas (EGA) (Lam, 2002a) as a new trend of cartography applied to teach school geography. We have been presenting theoretic (Xalisev, 2006) and practical approaches to design and edit (Lam, 2002b) a version of EGA for Thai Nguyen province with the purpose of teaching Geography in 9th and 12th classes (La et al., 2007 ).
\end{abstract}

\section{INTRODUCTION}

Map is a visual reflecting tool, modelizing real world, so map was used in many fields of social life for long time. When computers with specialized software, especially GIS were taken into map industry, a new type of map was introduced - digital map- with new concepts such as "layer", "subject", "features" and so on. The digital map is of advantages in showing, designing, establishing as well as being upgraded in appearance, load, flexibility, updatability and so on. This is an important premise to build and use the map

\section{BASIS TO DESIGN ELECTRONIC ATLAS}

Atlas- map set- system of maps with organic, accordant, supplemental and comparison relations for a unified form. Atlas is a map product with high scale and value(Lam, 2002a). Atlas has high value not only because of it quantity but also in its reasonable selection and meaningful arrangement. Thanks to systematic feature, it can be fully, comprehensively referred in an area, a certain matter, thus value of Atlas prevails over that of loose maps in Atlas in total.

With formation and development of digital map technology, Atlas also has break-through steps with appearance of Electronic Atlas. The term "Electronic Atlas" (E.Atlas) was first given by the author Eva Suekierska in 1984 and accepted for use by ICA World Map Association. Till now, some proposals have been given to use the term digital Atlas, atlas information system, cybercartographic atlas, but in general although the term E.Atlas has not shown full meaning and nature of the subject, it is still popularly used. The simplest way to understand E. Atlas is that Atlas is mainly formed and deployed in computer.

Based on achievements of information technology, digital map technology, E. Atlas brings advantageous functions compared to traditional Atlat on quality as well as way of formation and deployment. Canada, Switzerland, Holand and then America, Russia, China, Filand and so on are countries early applying digital map technology to build E. Atlas. This trend has been domestically developing with formation of a series of projects on E. Atlas both in national and regional scales (Vietnam Geography Atlas", "Vietnam's environment and natural resources Atlas", "Vietnam National Atlas", ”Dong Nai Electronic Atlas", " Ho Chi Minh city Electronic Atlas" and so on). However, design of electronic Atlas used in teaching local geography has not been built by any province.

\subsection{General requirements for textbook E. Atlas collection}

Geography Atlas is systematic collection of geographic maps built under a general outline as a complete work. Maps have organic and supplemental relations regulated by use purpose and characteristics in reality.

Textbook Atlas, also called textbook map collection, is a systematic collection of geographic maps, logically arranged for teaching purpose. It has high consistence on mathematics, contents and map outline. The textbook Atlas is distinguished with cover of territory, contents and use purpose.

General requirements for textbook Atlas collection include: (1) Each Atlas collection is compiled on base of one or several certain geographic programs. Thus, contents of maps must cover geographic contents of the whole program. In addition, due to systematic and complete features of Atlas collection, maps must fully specify all matters given in the theme, purpose and task of Atlas; (2) Internal consistent feature in the textbook Atlas is shown in reciprocal, supplemental relations with suitability and comparison among maps in Atlas. Purpose of the textbook Atlas is to ensure accurate reflection of supplemental relations and co-existing rules among phenomenon which the map system in Atlas should reflect, helping use of Atlas convenient and easy to withdraw accurate conclusions. Internal unification and suitability are most important, most complex during compiling contents of the textbook Atlas.

E. Atlas must comply with classification principles of Atlas in general (Nguyen, 2008), including:

According to cover of territory such as world Atlas, national Atlas, national-regional Atlas; provincial Atlas, district Atlas. According to purpose such as general geography Atlas; natural geography Atlas, common geography Atlas and so on. According to size: large size Atlas, medium size Atlas, small size Atlas and so on.

In addition, for E. Atlas, it can be distinguished according to interaction levels and ability to carry out analysis during exploitation process:

"View-only" Atlas: User only views available maps (often in bitmap). This type of Atlas is like traditional Atlas, only different in opening on computer, room-in, room-out, random extract to a certain content, map instead of orderly opening as on paper. 
"Interactive" Atlas: In this Atlas, ability to display information is more flexible, user can act some processing movements with map to change mode of display (re-select color mode, way of classifying data and so on) appropriate to objective desire.

"Analytical" Atlas: supply most GIS analysis functions to reply not only questions "Where? What?" but also reply questions "How?" and "Why?" through space analysis method provided. To ensure high internal unified feature, when designing E. Atlas, take notes:

Pursuant to theme, use purpose and Atlas drawing territory to select suitable reference methods (Pham, 2008), number of references should not be redundant. Order to arrange map group and maps is based on the principle from general to particular, natural to economic.

Design of map contents is based on a unified principle. Same type phenomenon shown on different maps must use same method and unified display criteria. Quickly changed geographic contents should use unified data in a same certain period of time to compare.

Generalization principle of map in Atlas must be consistent from first to final maps. It is the unification in generalizing contents, classification, decentralization of quality criteria and quantity indexes must unify in annotation.

Use the unified method on maps. It is to use the unified color, sign, and font for same subjects.

Using unified geographic basis is important condition to ensure internal unification in Atlas.

Map updating is an important standard to ensure quality of a Atlas collection. Latest data source helps formation of map gain updating standard of Atlas. Each Atlas collection must fully ensure scientific, visual and pedagogical features of the textbook map collection.

\section{DESIGN AND COMPILE GEOGRAPHY E. ATLAS OF THAI NGUYEN PROVINCE}

\subsection{Formation process of E. Atlas of Thai Nguyen province}

Atlas is built under following steps (Nguyen, 2007) : design, prepare to compile: compose general design outline, then outline for each chapter, moderlize, build database; finally compose outline for each map. Compiling process: edit map on computer, possibly try many options. Prepare to print: Separate electronic color with optional number of color separation films, not depending on number of colors; reducing many intermediate products and giving accurate results. Publish under many diversified forms: print on paper, numeric form on disc or network. Implementation time is shortened much in comparison with formation of traditional Atlas because materials should not be much prepared. However, equipment should be newly invested because software for program requires high and modern configuration. Personnel should be trained. Quality of product is high and consistent because it is built on computer. Ability to repair is simple, regularly updated.

Thai Nguyen Atlas is selected to design in Powerpoint 2003. This is a "active" and "open" Atlas, formed on base of software ArcGis 9.2, Mapinfo 9.0 and Powerpoint to display maps. After formation and compiling the complete Thai Nguyen Atlas on ARCGIS 9.2, use Powerpoint 2003 to design Thai Nguyen electronic Atlas.
Each map is a slide arranged under from general to concrete order and maps have organic relations together. The electronic Atlas can be recorded in USB orderly and conveniently for display, change, especially most high schools are equipped with hi-tech rooms, modern showing devices, ADSL broadband connection and so on.

To easily use, possibly regulate how to transmit for each slide or at the same time for all slides of an browser. On Menu taskbar, select Slide show, Slide Transition, default select No Transition (Modify: select Fast- slide show- fast movement; advance - On mouse click- default when clicking mouse, finish slide show. Select automatically after 2 seconds: automatically finish after 2 seconds. Click to select Apply to all to apply transition mode for all slides of map collection in Atlas.

Possibly establish foundation for map slide, or use available Template. When selecting available Template, for each use of command Insert/ New Slide, new Slide still follows this Template. Thus, Atlas collection will have same foundation color.

Thai Nguyen province geographic (?) E. Atlas has many flexible layers. Possibly change mode, rate, and vision as required, being supported by Multimedia. Extract ability is fast as required. Have tools to automatically make statistics, analyze, measure. Require user to have computer using skills. Ability to update: quickly and easily conduct each part. Quickly establish the implementation steps with stable quality of product. Invest in devices and training of personnel. Publish under diversified and flexible forms such as paper, file, HTML, possibly extract each part with low price compared to publishing of paper Atlas collection.

\subsection{Introduction of Thai Nguyen province E. Atlas}

\section{BẢN ĐỐ HÀNH CHÍNH TİNH THÁI NGUYÊN}

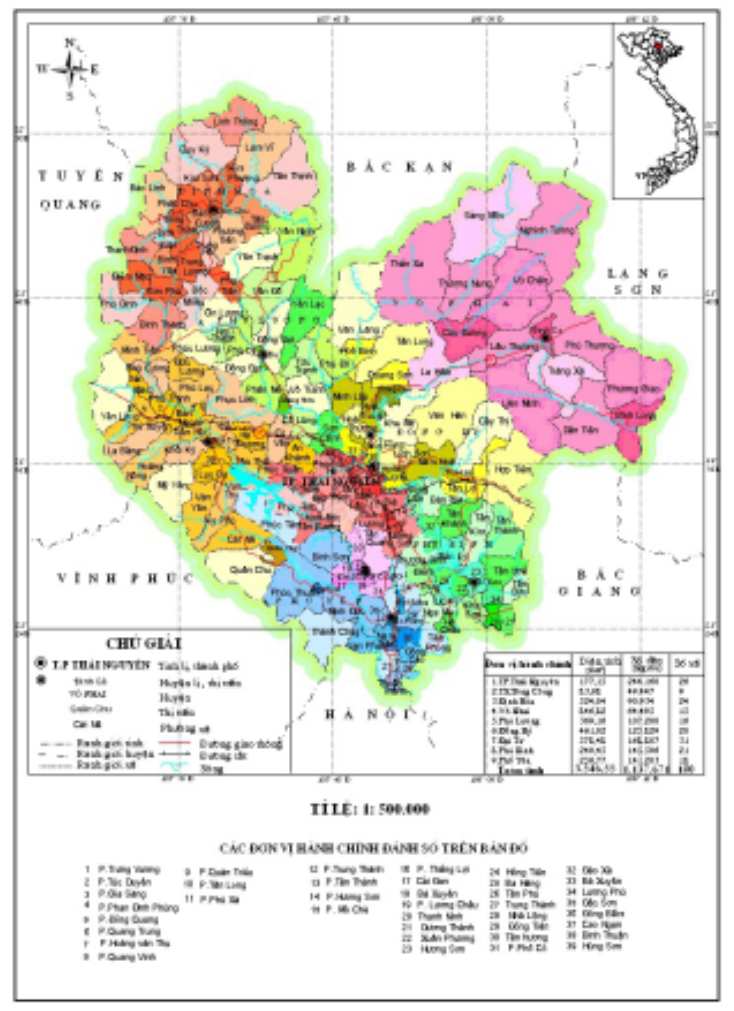

Figure 1: Administration E.Atlas of Thai Nguyen Povince

Atlas includes official map pages and is arranged as follows: 
a. General map pages: reference grid, rate, general signs and some typical topography forms of Thai Nguyen province shown with overview features on geographic location, territory scope of Thai Nguyen province (?) ; page "Thai Nguyen province in nature of the North"; page "Thai Nguyen province in Northern provinces".

b. Map pages of natural geography include: Thai Nguyen topography map: presenting some typical topography forms; geological map and mineral of Thai Nguyen province: fuel minerals, metal minerals, non-metal minerals, construction material minerals. Showing methods: Geology (quality foundation method); mineral (signing method). Climate map of Thai Nguyen province includes following contents: climate areas shown by quality foundation method, annual changes of temperature, rainfall by the locating diagram method, types of wind to Thai Nguyen province by moving sign; particularly temperature and rainfall maps shown by adequation method combined with coloring under layer. Contents on soil, plant and animals of Thai Nguyen province include maps: soil map shows key types of soil in Thai Nguyen province by the quality foundation method. Animal and plant map shows types of key plants and animals under water and on land by allocation method.

c. Social map pages include: map of community of Thai Nguyen provincial ethnics shows ethnics living in Thai Nguyen province by the quality foundation method. Residential allocation map shows allocation of population in urban, rural areas, ethnic components and density of the province by marking method. Population density is allocated under commune level. This content has important meaning in determining residential allocation in commune territory. The method used to show residential density is diagram method. Administrative border is defined under commune level and through statistic data table to give density scale. Auxiliary content: map showing population of Thai Nguyen province through years from 2002 to 2007, map of population increase rate of the province period 2002- 2007, map of urban and rural population structure in 2007, map of provincial ethnics structure in 2007. Each resident map page arranges some (?) necessary diagrams to explain key contents on these maps. In addition, some images of typical ethnics in Thai Nguyen province are also explained.

d. Pages of agricultural- forestry- aquatic economic map include general agriculture map showing agricultural areas by the quality foundation method and allocation area method. Contents: Reality of land use; key features of agricultural production (scale and distribution of plants; scale and distribution of pests; natural forest and planted forest). Some auxiliary contents: diagram of agricultural-forestry- aquatic structure; production value diagram of agricultural sector structure in 2007; rice output diagram through years from 2003 to 2007; tea output diagram through years from 2003 to 2007. Some other maps: Map of planting foodstuff plants, short-day and long-day industrial trees, vegetables (?) , rearing map (big cattle, small cattle and livestock). Map of reality of Thai Nguyen province forest allocation shows by the allocation area method. Fish-breeding map shows types of fishes, key rivers with economic value in the province by allocation area method and line signs.

e. Pages of industrial economic map include: Industrial map (including local and national industrial sectors) showing big, medium and small industrial centers, key industrial sectors by the signing method. Small-scale industrial map (traditional village and fine art facilities).Auxiliary contents con-

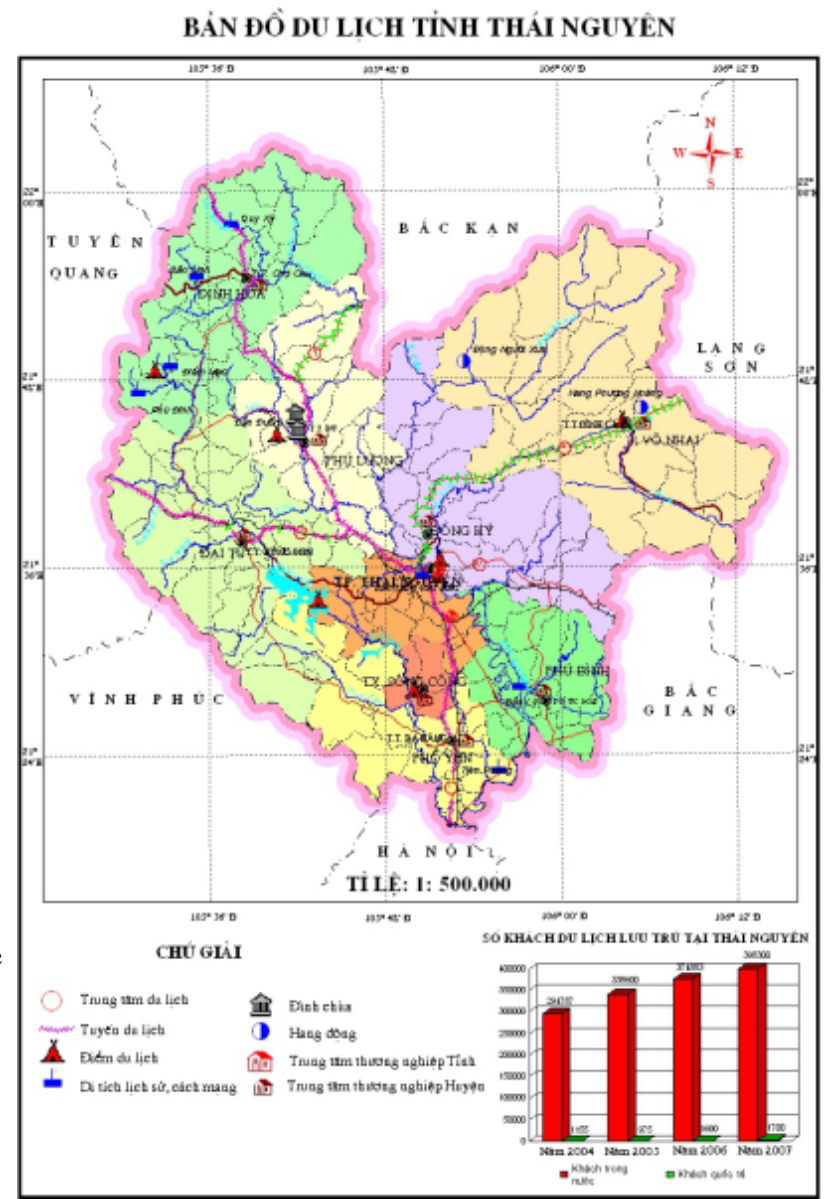

Figure 2: Tourist E.Atlas of Thai Nguyen province

sist of map of industrial value through years 2002- 2007; map of industrial production value in the province in accordance with industrial sector; industrial production map exploited through years; processing industrial production map through years.

f. Pages of service map: Transportation map; commercial map and tourism map of Thai Nguyen province are shown by the signing method, signing under line, movement, Cartodiagram and Catorgram.

g. Map of economic zone is designed with diagrams to clearly explain key contents shown on maps. Auxiliary contents include map of import- export turn-over through years; map of transited quantity of goods; map of number of transited passengers through years; Map of commercial and service facilities.

h. Maps on environment are built to help pupils find that in addition to sustainable economic development, protection of local natural resources and environment cannot be ignored.

\section{CONCLUSIONS}

Thai Nguyen province geography E. Atlas is built to teach and learn local geography program in high schools, equip and strengthen knowledge on map, local geography for pupils. Through map, pupils understand signs, map showing method, recognize contents on map, analyze, synthesize and withdraw conclusions on geographic subjects. 
Thai Nguyen province geography E. Atlas is built and used in "Local Geography" program of 9th and 12th classes, focusing on some contents on: geographic location and distribution of administrative units, natural conditions and natural resources, resident and labor force, geography of industrial, agricultural, service sectors and so on. With contents and way of showing Atlas by PowerPoint as designed, Thai Nguyen province geography E. Atlas will be useful for teachers and pupils. Teachers should thoroughly exploit knowledge in E. Atlas to mobilize effects of Atlas. However, depending on each contents of the lesson, contents of map in E. Atlas combined, select suitable method to bring high efficiency, mobilize positive feature and self-occupy knowledge of learners.

E. Atlas with predominant functions compared to traditional Atlas is an explanation, an open way for the matter given. Establishment of Thai Nguyen E. Atlas makes an important contribution to local geography teaching and learning method in the province in current period. The above theoretical basic can be applied generally to socio - economic thematic mapping, preferable in digital technology. The guideline for establishment atlas will be applied well , therefore, this guideline could be used as reference for other coming projects.

\section{ACKNOWLEDGEMENTS}

The authors would like to thank the Thainguyen statistic office in Vietnam, Associate professor Dr Lam Quang Doc- Geography Department in Hanoi National University of Education, Dr Vu Nhu Van-Geography Department,Thai Nguyen University of Education. Thanks also go to the the reviewers for their valuable comments

\section{REFERENCES}

Lam, Q.D. 2003. Cartography. Vietnam Education Publishing House, pp. 199-257.

Lam, Q.D. 2002. Theme Map. Vietnam Education Publishing House, pp. 200-209.

Lam, Q.D. 2002. Theme Map. Vietnam Education Publishing House, pp. 211-213.

Xalisev, K.A. 2006. Cartography. National Hanoi University Publishing House, Editing in 2006, pp. 254-302.

Le, D.H., Do, X.S. 2008. Applying database building geographic information system and map collection of natural conditions, natural resources and environment of Hanoi city. Proceedings of the rd National science conference on geography, pp. 968-975.

Nguyen, Q.T. 2007. Vietnam Geography Atlas. Vietnam Education Publishing House, Hanoi.

La,T.L; Nguyen, V.T; and Lam, Q.D 2007 Knowledge exploitation in Vietnam Geograpgy Atlat for teaching and learning in 12th class. Hanoi Pedagogy University Science Magazine,

Thai Nguyen statistic office, 2006. Thai Nguyen statistic year book. Thai Nguyen publishing House

Thai Nguyen statistic office, 2007. Thai Nguyen statistic year book. Thai Nguyen publishing House

Nguyen, T.T., 2000. GIS organization. Construction Publishing House, Hanoi
Nguyen, C.V, 2008. Scientific basis to build electronic map collections. Proceedings of the 3rd National science conference on geography, pp. 952-958.

Pham, Q.V., 2008. Applying combination of digital image processing method and Geographic information system (GIS) in building theme map. Proceedings of the 3rd National science conference on geography, pp. 985-987.

GIS software, Mapinfo 9.0. 